\title{
iFace Identification and Authentication Technology Based Collaborative Effort to Resolve the Security Problems
}

\author{
Mehran Sayed, Arifa Bhutto, Arjumand Bano and Imran Anwar Ujan \\ Institute of Information and CommunicationTechnology, University of Sindh, Jamshoro, Sindh, Pakistan; \\ mehransayed92@gmail.com, arifa.bhutto@usindh.edu.pk, arjumand@usindh.edu.pk, \\ Imran.ujan@usindh.edu.pk
}

\begin{abstract}
Main goal of this research paper, we stated that bio-metric and eye face identification and authentication of a human face. It highlights mouth, nose and upper part of face it is very sensitive technology, if a candidate stands in a different moods of facerather than the natural human face expression e.g. happy, angry, sad, etc. the system would not be able to identify their facial expression and finally the entity itself. The literature in the reference sections suggests that this area of research is gaining more customers in terms of organization and their concerns. Pakistan is one of the targetedareas of the different civilizations and people. It is very hard to handle anyone of them have the ill-will. UsingFace recognition algorithm, Eye Face type biometric machines can capture from the distance of less than one meter it controls and easy to locate a person identification. It is reliable technology and fast to identify candidate information, it is used for security purposes in organizations such as E-passport site, Banks automatic transition and authentication of person.
\end{abstract}

Keywords: Algorithm, Authentication, Bio-metric, iFace, Recognition

\section{Introduction}

Bio-metric and iFace Identification Technology is based to identify candidate which works in organization for the security purposes that he/she is original employee of organization and check their daily attendance in which time candidate check in and out. There is sustained and collaborative effort or resolve the security problems. Our research is based on iFace and bio-metric recognition application design algorithms and integrated with the hardware we design the application which resolve the real time face identification problem. iFace identification and bio-metric authentication that is based on iris recognition system, nose (e.g. half of the face), thumb and fingers based application. That means machine recognizes a candidate and identify information.

Bio-metric and iFace identification technology is based to identify candidate which works in organization for the security purposes that he/she is the original employee of organization and check his/her daily attendance in which candidate checks-in and checks-out. The goals of the sustainable development and meeting the human and natural systems to cope with the natural and ecosystem services and to help this system gain the goals of the sustainable development the end result is the living conditions would improve through this system that is to help check the premises according to the security and activities which is of the prime objectives. This system not only helps the logged in activities but also help organization to economically secure system more then the human based security system which is required more labour and less secure.

The modern era of the Pakistan is to develop and provide a sense of security in terms of handshaking with the super powers like China and other countries. The system is based on computers that are not gaining more electricity hence environmental protection is also taken care of by using that system.

*Author for correspondence 
The face detection and recognition is a creative approach, whichdetect, scan the image, fingerprints, and using a high level coding to detect the object and shows the better result of facial expressions and match the images one is stored image in database through machine and other the front face of person.

Number of Governmental and Non-governmental organizations in our country Pakistan needs the systems that can not only check the entrance and exit of the concerned organizations but also to check the activity of their employees who are working in their neighborhood. Such number is huge in the organizations of the Pakistan that require proper monitoring and control on who is currently in the office environment to safeguard the human lives in the event of a disaster.

The aim of research the system will help to monitor the entry and exit of the people in the organization and manage the pay roll system, security system who deals with bio-metric system and face identification and attendance system. Our country has less resource in terms of the coping with the problems of the terrorism activities, energy crises, educational system, unemployment, political system, environmental, and others. One of the major is the security and law enforcement that can be handled by the accurate check and balance of every person comes in and goes out of the organization. This system is the solution.

There are three approaches of facial recognition:

1. Hybrid approach, 2. Feature base method approach,

3. Holistic approach

Hybrid approach: In this approach the whole face is detected and it is the combination of holistic and feature approaches.

Feature base method approach: In this approach the face is divided into parts like mouth, nose and eyes and get the input values of face detection and easy to recognize the face. It segments the face and detect the different part of face and easy to recognize faces.

Holistic approach: This approach defines the whole face detection get inputs of face and system recognizes the face.

\section{Biometric Applications and Systems}

Today bio-metric and face recognition application widely used for the sack of security issues and also controlling the threats of terrorist using smart cameras to detect the face and recognize easily to provide the data of person from the database then we can control easily unknown person. Mostly these systems used in airports, hospitals, government departments, schools and other private departments to verify their workers using face detection and bio-metric systems.

\section{Face Recognition and Identification}

The face recognition is a technique which identify the persons face, which is stored in database when the face will appear in front of system the identification process will start and recognize that person from given data.

The flow chart describes the face recognition as shown in Figure 1.

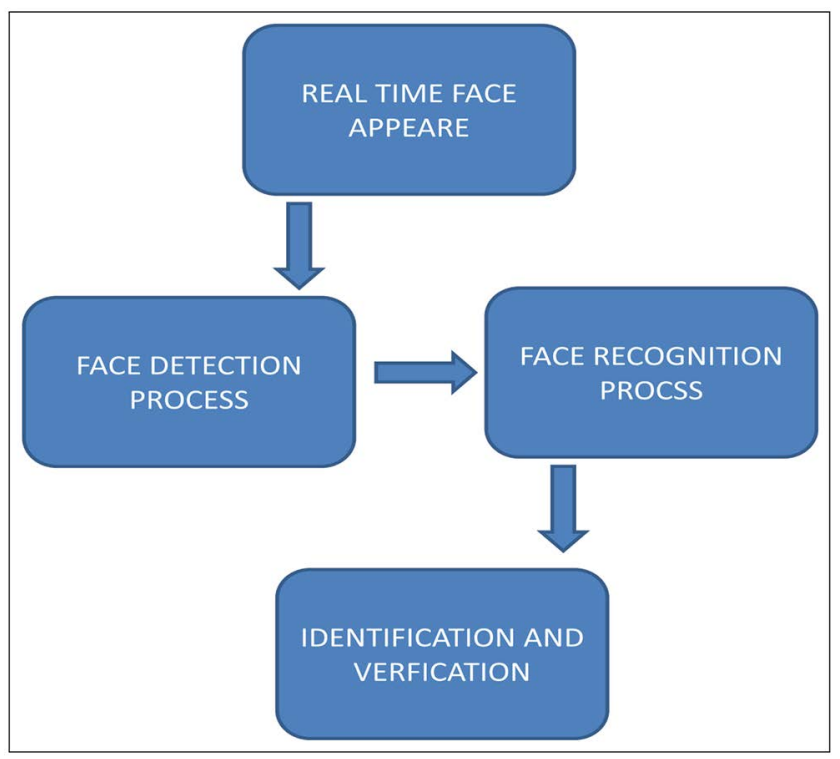

Figure 1. Face identification and verification.

\section{Related Work}

Over the last forty years the research of face recognition is ongoing there are several approaches and methods are using in different researches such as machine learning, image processing, neural network, computer vision, recognition pattern, face identification and authentication, face detection algorithms, face recognition algorithms and many other approaches are using to solve the face expression and identify in easiest way because there 
is a problem in identification and recognition system with the different expressions of human face and the machines could not identify the mode of human face but the classification is the main problem, human is the best solution to recognition technology but here is a limited memory problem. So the problem solution there is the huge work on machine learning languages to easy recognize the human face, here is some problems to face the machine in recognition and identification of human face that are:

1. Changing face expressions.

2. Face changing with time.

3. Human age problem of face.

4. Direction changes.

5. Geometric scaling.

6. Stored Profile and Frontal face.

7. The presence of mustaches.

8. Absence of beard.

9. Absence of mustaches.

10. Wear mask.

11. Wear scarf in front of face.

12. Presence of beard.

The automatic recognition of face is the complicated approach is involved which is face detection techniques, the facial recognition and face feature extraction. Face recognition algorithm which is divided into two sub classes to solve the problem one is image templet and feature based. The image based template defines the correlation among the image and image template which is stored in database ${ }^{1}$, Optimal strategy faces recognition ${ }^{2}$, Support vector machines ${ }^{3}$, Iris recognition ${ }^{4}$, In security technology dyadic wavelet transform zero-crossing algorithm using identification and recognition ${ }^{4}$, Pattern recognition workshop ${ }^{5}$, Biometric introduction ${ }^{6}$, Iris identification ${ }^{7}$. Digital watermarking to protecting iris images ${ }^{\circ}$, Linear analysis ${ }^{11}$, Kernel approach $\frac{12}{}$, Image template saving suitable database $\mathrm{e}^{\underline{13}}$, Face verification results ${ }^{\underline{11}}$. Quality matric based comparators iris technology?: Non cooperative iris and biometric recognition ${ }^{10}$, Automatic human face recognition $\frac{12}{}$, Face detection feature ${ }^{13}$. Rotated face detection algorithm ${ }^{\frac{14}{4}}$, Attacks on machine learning models ${ }^{15}$, Attacks on deep learning models ${ }^{16}$, Object detection and semantic segmentation ${ }^{17}$, Neural network evaluates the robustness in privacy and security problems $s^{\frac{18}{}}$, Assessment of image quality $\frac{19}{}$, Defense competition and attacks $^{20}$.

\section{Research Design}

In research model, we use a camera to detect the real human face and recognizer to recognize the face parts, which are mouth, nose and upper part of face, put the correct information of employee, and store it into database. This is easy way to identification of employee's with face recognition technique, and also generate the many dynamic reports of employee monthly and daily reports of attendance and many other reports. For security purpose we using SQL Server database system which is secure and easy to use to manage the data information and in GUI we use $\mathrm{C}$ sharp language easy to connect face recognition machine with IP address, control machine settings store employee information from system to machine and get the daily attendance logs generation daily attendance reports in CSV format. Here is a research model define in Figure 2.

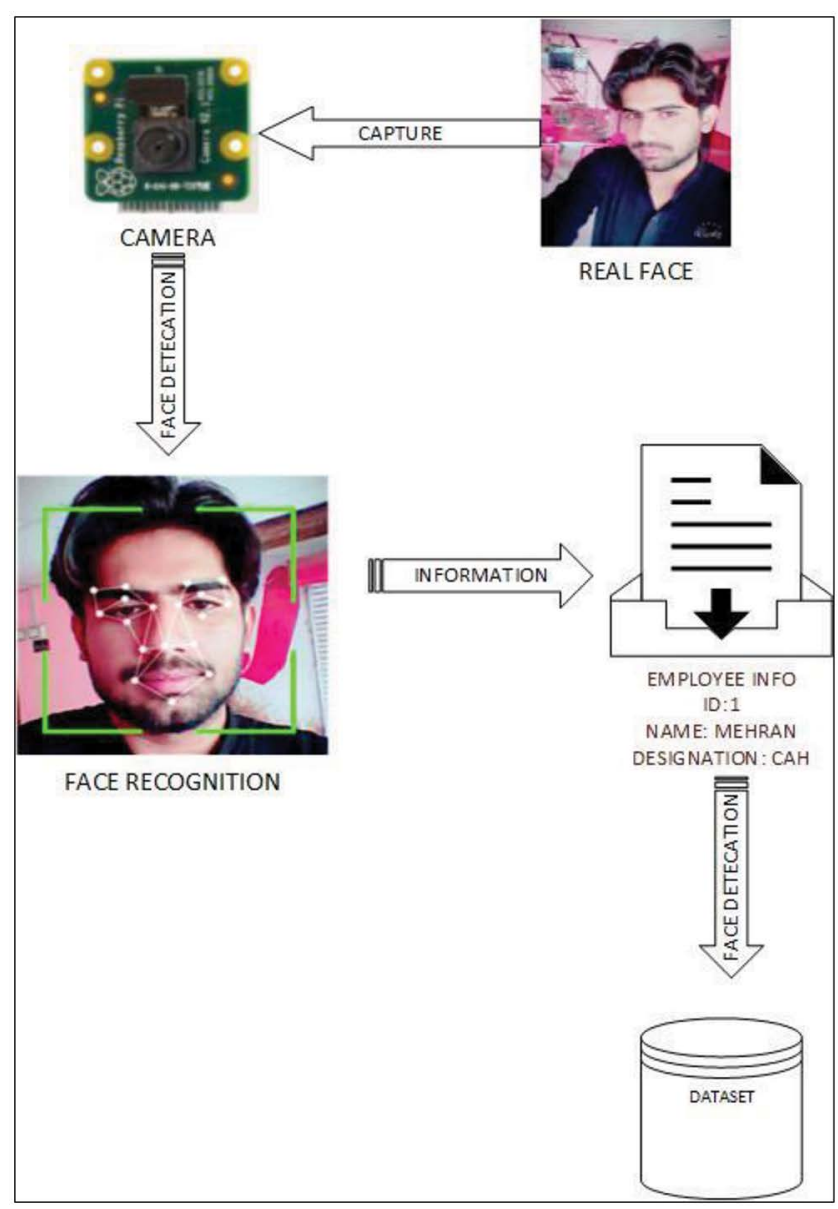

Figure 2. Research design. 


\section{Research Methodology}

In this research article we implemented the recognition algorithm to detect the face verification and identification and generate the daily attendance reports and automatically logs save into database. Actually we developed and implement software in government and private sectors to detect the actual employee of company for security purposes and daily attendance and save it into database and generate the daily attendance reports into CSV files and also it saves the employee data and handle the pay role system we are using C\# Language to develop the system and easy to connect face recognition machines and using SQL Server 2016 to store database of employees in Figure 3.

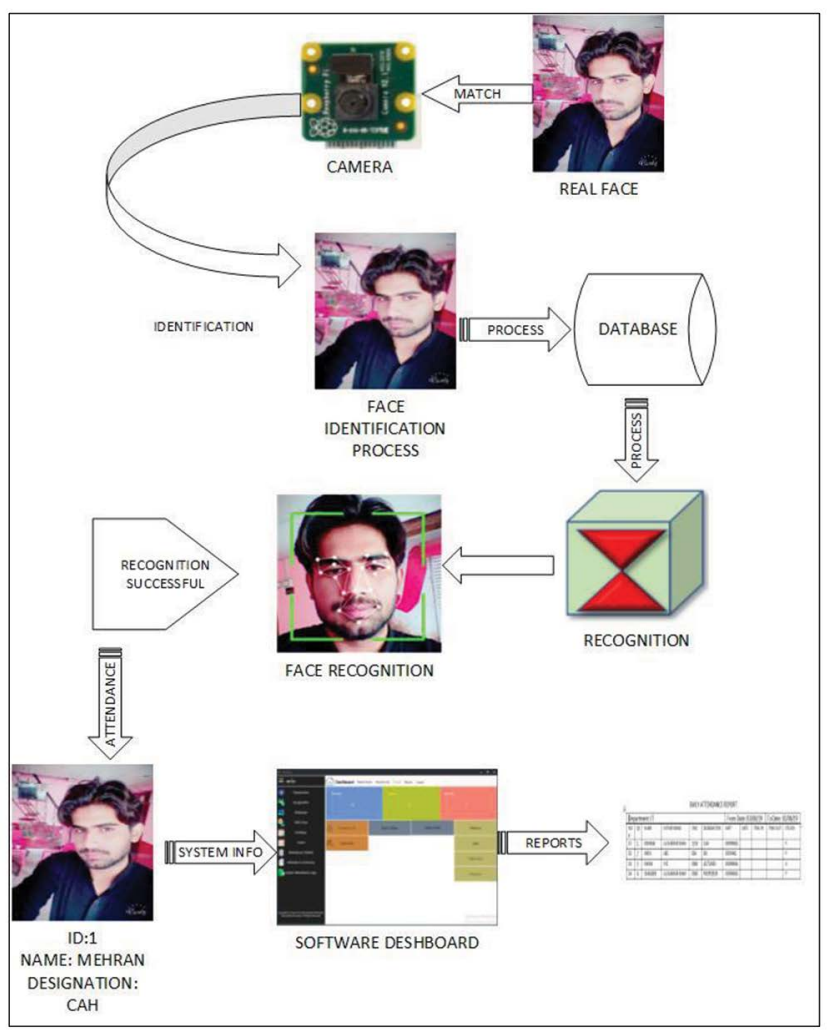

Figure 3. Research methodology.

\subsection{Recognition of Facial Expressions}

The face expression is based on face detection technique. In this paper we describe the recognition of face expression. Face recognition as shown in Figure 4.

Figure 4 showing the seven different modules. First module describes the video camera to capture the image.

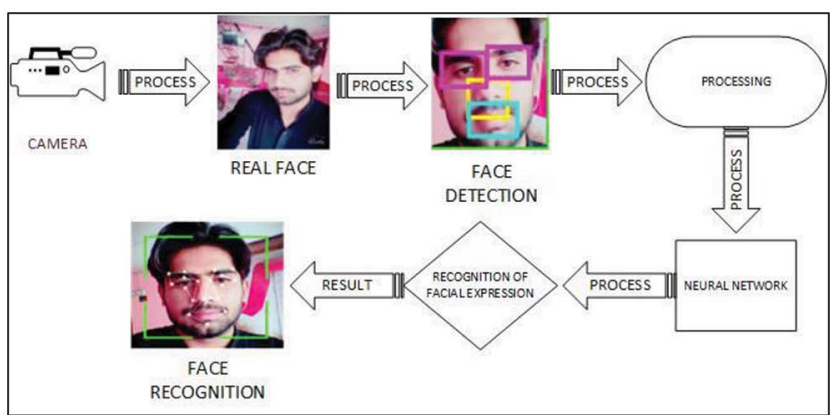

Figure 4. Face recognition architecture.

Second capture the image third module defines the detected face from capture image this module is covered the bounding box on image and show the blocks on face different parts such as mouth, nose, left eye and right eye and cover the whole face with boundary box. Describe the thinning, generation of tokens, histogram equation and edges of detection. Fourth module define the information to other fifth module to processing the neural network the six one describe the recognition of face expression and the last one is the result of face recognition

\section{Face Detection and Recognition Steps}

The facial expressions to detect and recognition, identify the persons face, which is stored in database when the face will appear in front of system the identification process will start and recognize that person from given data.

Face recognition algorithm is defines the following steps and shown in Figure 6.

1. Add image.

2. Face detector objects.

3. Using face detector objects on image.

4. Insert image in database.

Comparing the images stored image and input image.

1. Insert image.

2. Face detector object.

3. Using face detector objects on image to extract the face features and detect the face.

4. Match the image with database image.

Testing RESULT of input image and stored image the process of face recognition shown in Figure 5\& 6 . 


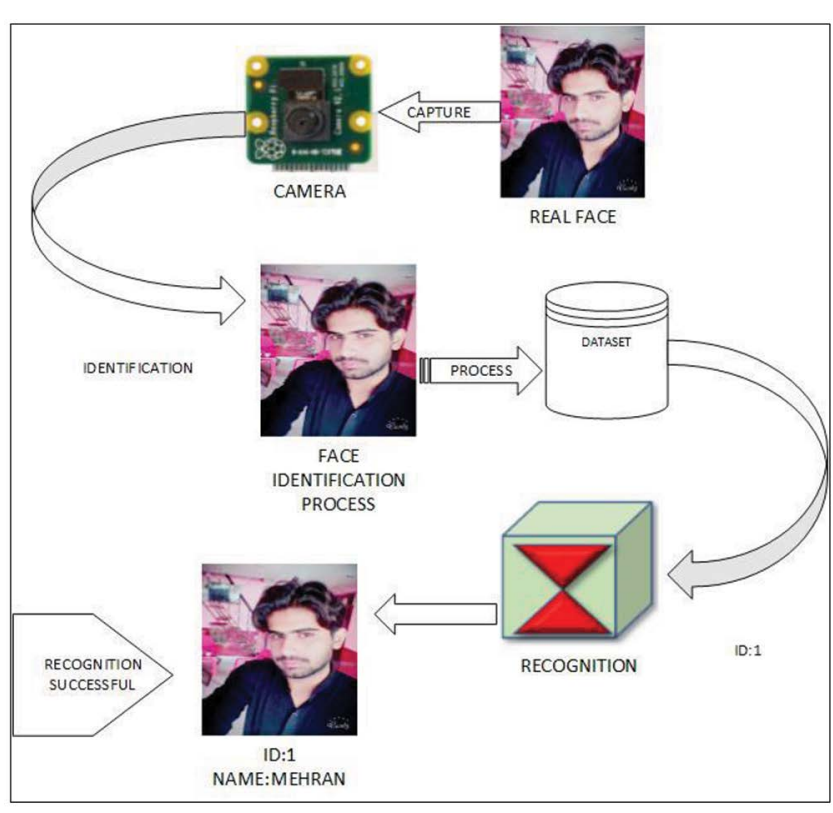

Figure 5. Successful result of face recognition.

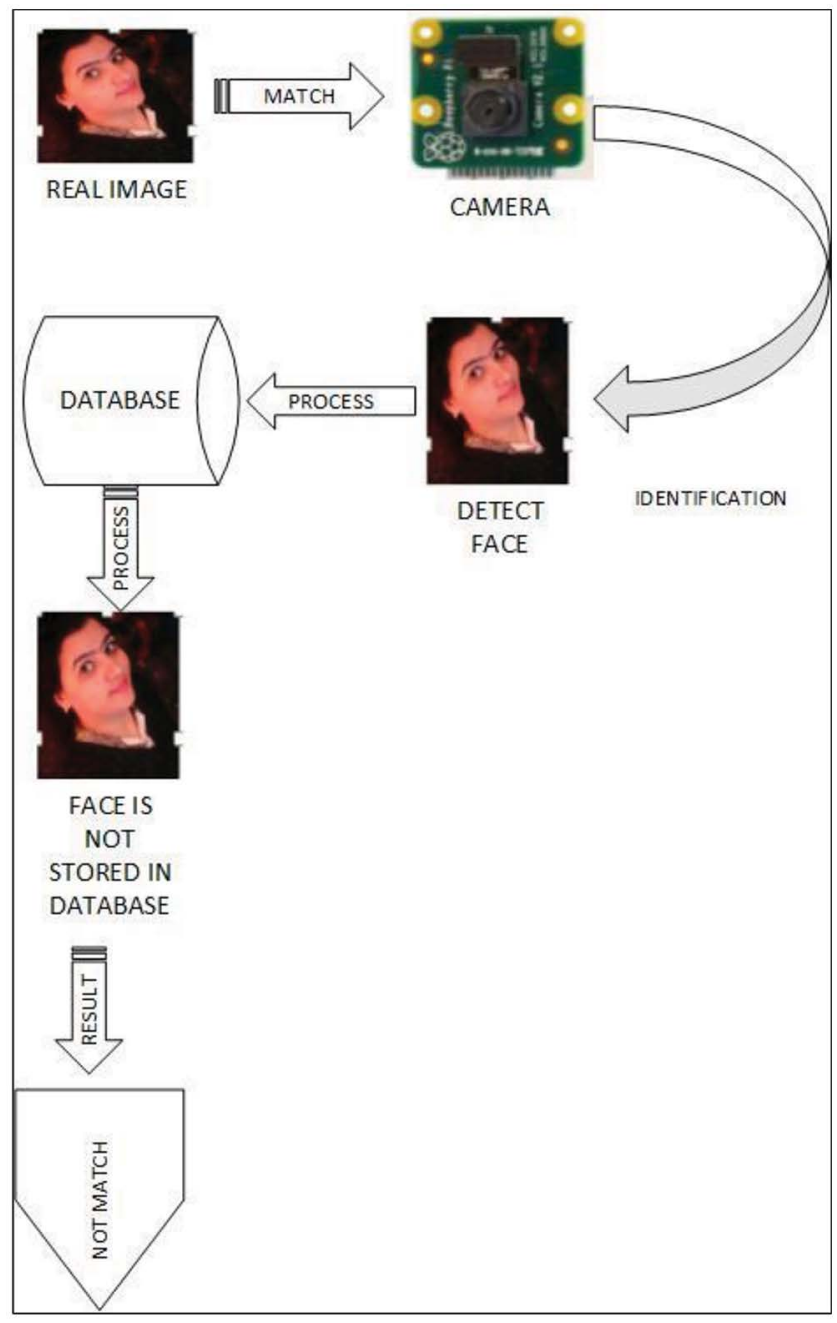

Figure 6. Unsuccessful result of face recognition.

\section{Conclusion}

In this research paper, we focused on iFace Identification and Authentication Technology sustained and collaborative effort or resolve the security problems using face recognition algorithm to detect the face and match with database image and get the results of that person. There are many different application on face recognition are available but still lots of work needed to be done in term of the security of the information is concerned. In our design application,we developed the algorithm that directly detect the face of the employee and connected with the employee databases, which also connected with the payroll system of the organization. Our designed system connect the many different face, biometric machines that, and set password on machinegenerated everyday logs from machine and set the many other activities. We provide easy and secure user interface with admin authentication using C\# Language to create application and for store information we using SQL Server 2016 database.

\section{References}

1. Hallinan PW. Recognizing human eyes. In: SPIE, Proceedings Volume 1570, Geometric Methods in Computer Vision, San Diego, '91, 1991, San Diego, CA, United States; Sep 1991. p. 214-26. https://doi.org/10.1117/12.48426.

2. Karthikeyan T. Efficient bio metric iris recognition system using fuzzy neural network, International Journal of Advanced Networking and Applications, 2010; 1(06); 37176. https://pdfs.semanticscholar.org/5066/66be9b5b9fcab2 fc670f438ff37c1e90719e.pdf.

3. Ouda O, Tsumura N, Nakaguchi T. Tokenless Cancelable Biometrics Scheme for Protecting Iris Codes. In: Pattern Recognition (ICPR), 20th International Conference, IEEE, Istanbul, Turkey; 2010, p. 882-85. https://doi.org/10.1109/ ICPR.2010.222.

4. de Martin-Roche D, Sanchez-Avila C, Sanchez-Reillo R. Iris Recognition for Biometric Identification using Dyadic Wavelet Transform Zero-Crossing. In: Proceedings IEEE 35th Annual 2001 International Carnahan Conference on Security Technology (Cat. No.01CH37186), London, England, UK; 2001. p. 272-77. doi: 10.1109/ CCST.2001.962844.

5. Rathgeb C, Uhl A. Statistical Attack Against Iris-Biometric Fuzzy Commitment Schemes. In: Computer Vision and Pattern Recognition Workshops (CVPRW), IEEE Computer Society Conference, Colorado Springs, CO, USA; 2011. p. 23-30. https://doi.org/10.1109/CVPRW.2011.5981720. 
6. Jain AK, Ross A, Prabhakar S. An introduction to biometric recognition, IEEE Transactions on Circuits and Systems for Video Technology. 2004; 14(1):4-20. https://doi. org/10.1109/TCSVT.2003.818349.

7. Noh SI, Pae K, Lee C, Kim J. Multiresolution independent component analysis for its iris identification. In: Proceedings ITC-CSCC: International Technical Conference on Circuits Systems, Computers and Communications, Phuket Acadia Hotel \& Resort, Phuket, Thailand; 2002. p. 1675-78. www. comlab-gist.kr/NFUpload/nfupload_down.php?tmp_ name...2400.7.0...6...

8. Bartlow N, Kalka N, Cukic B, Ross A. Protecting iris images through asymmetric digital watermarking. In: IEEE Workshop on Automatic Identification Advanced Technologies, Alghero; 2007. p. 192-97. https://doi. org/10.1109/AUTOID.2007.380618.

9. Hofbauer $\mathrm{H}$, Rathgeb $\mathrm{C}$, Uhl A, Wild P. Iris recognition in image domain: Quality-metric based comparators. In: Bebis G. et al. (eds) Advances in Visual Computing. International Symposium on Visual Computing, Lecture Notes in Computer Science, Vol 7432, Springer, Berlin, Heidelberg; 2012. p. 1-10. https://doi.org/10.1007/978-3642-33191-6_1.

10. Colores JM, García-Vázquez M, Ramírez-Acosta A, PérezMeana H. Iris image evaluation for non-cooperative biometric iris recognition system. In: Batyrshin I., Sidorov G. (eds) Advances in Soft Computing. MICAI 2011. Lecture Notes in Computer Science, vol 7095. Springer, Berlin, Heidelberg; 2011. p. 499-509. https://doi.org/10.1007/9783-642-25330-0_44.

11. Matas J.-Hamous, M-Jonsson. Comparison of Face Verification Results on the XM2VTS Database. Proceedings of the International Conference on Pattern Recognition, Vol 4; 2000. p. 858-63. https://dl.acm.org/citation. cfm?id=877771.

12. Jie Z -Chunyu, L -Changshui Z. et al. A Survey of Automatic Human Face Recognition, Acta Electronica Sinica. 2000; 28(4):102-06. http://en.cnki.com.cn/Article_en/ CJFDTOTAL-DZXU200004026.htm.

13. Jeng Shi -H, Liao Hong -Y. Mark, et al. Facial Feature Detection Using Geometrical Face Model: An Efficient
Approach, Pattern Recognition. 1998; 31(3):273-82. https:// doi.org/10.1016/S0031-3203(97)00048-4.

14. Lv X.-Jie, Z.-Zhang C. A Novel Algorithm for Rotated Human Face Detection. Proceedings of the International Conference on Computer Vision and Pattern Recognition, IEEE Computer Society Conference on Computer Vision and Pattern Recognition, vol. 1; 2000. p. 760-65. https:// www.researchgate.net/publication/3854192_A_novel_ algorithm_for_rotated_human_face_detection.

15. Shokri R, Stronati M, Song C, Shmatikov V. Membership Inference Attacks Against Machine Learning Models, In: IEEE Symposium on Security and Privacy (SP), San Jose, CA; 2017. p. 3-18. doi: 10.1109/SP.2017.41. https://doi. org/10.1109/SP.2017.41.

16. Evtimov I, Eykholt K, Fernandes E, Kohno T, Li B, Prakash A, Rahmati A, Song D. Robust: Physical-World Attacks on Deep Learning Models, Vol. 1., Cornell University; 2017. https://arxiv.org/abs/1707.08945.

17. Xie C, Wang J, Zhang Z, Zhou Y, Xie L, Yuille A. Adversarial Examples for Semantic Segmentation and Object Detection. In: IEEE International Conference on Computer Vision (ICCV), Venice; 2017. p. 1378-87. doi: 10.1109/ ICCV.2017.153. https://doi.org/10.1109/ICCV.2017.153. PMCid: PMC5492339.

18. Carlini N, Wagner D. Towards Evaluating the Robustness of Neural Networks, In: IEEE Symposium on Security and Privacy (SP), San Jose, CA; 2017. p. 39-57. doi: 10.1109/ SP.2017.49. https://doi.org/10.1109/SP.2017.49.

19. Flynn JR, Ward S, Abich J, Poole D. Image Quality Assessment Using the SSIM and the Just Noticeable Difference Paradigm, In: International Conference on Engineering Psychology and Cognitive Ergonomics. Springer, Berlin, Heidelberg; 2013. p. 23-30. https://doi. org/10.1007/978-3-642-39360-0_3.

20. Kurakin A, Goodfellow I, Bengio S, Dong Y, Liao F, Liang M, Pang T, Zhu J, Hu X, Xie C., et al. In: Escalera S., Weimer M. (eds), The NIPS ' 17 Competition: Building Intelligent Systems, The Springer Series on Challenges in Machine Learning, Cham; 2018. https://doi.org/10.1007/978-3-31994042-7_11. 